\title{
High-harmonic gyrotrons with irregular microwave systems
}

\author{
A.V. Savilov, I.V. Bandurkin, M.Yu. Glyavin, Yu.K. Kalynov, Yu.S. Oparina, I.V. Osharin, \\ N.A. Zavolsky
}

Institute of Applied Physics RAS, N. Novgorod, Russia, savilov@appl.sci-nnov.ru

A natural way to increase the gyrotron frequency is the use of operation at higher cyclotron harmonics. However, at moderate voltages, the intensity of the electron-wave coupling decreases rapidly with the increasing harmonic number. Due to this fact, the danger of excitation of parasitic close-to-cutoff waves at lower harmonics becomes a serious problem. This is true especially in low-relativistic gyrotrons with low output powers (and, therefore, low operating currents) at least due to two reasons. First, low-harmonic waves possess bigger electron-wave coupling factors. Second, longer operating cavities are required, in general, to excite high-harmonic operating waves. The latter results also in extremely high diffraction Qfactors for the operating near-cutoff waves and, therefore, a great share of ohmic losses. In this work, we describe several schemes of cavities with short irregularities designed to improve operation of lowrelativistic high-harmonic gyrotrons.

\section{Cavities with decreased ohmic losses}

A method to combine a long-length interaction region of a high-harmonic gyrotron and a relatively low diffraction $Q$ factor was proposed in Refs. [1,2]. This method is based on the use of a sectioned system (Fig. 1 a) with a klystron-like character of the electron-wave interaction: particles interact with a nearcutoff wave only in the input and output cavities (sections 1 and 3), whereas the drift region (section 2) provides coupling between these cavities as well as bunching of electrons (Fig. 1). The diffraction $Q$ factor in such a scheme is determined mainly by the sum of lengths of the two cavities, $Q_{\text {dif }} \propto\left(L_{1}+L_{3}\right)^{2}$. At the same time, the starting current is determined by the total length of the system, $\left(L_{1}+L_{2}+L_{3}\right)$.This scheme was realized in the pulsed large-orbit $80 \mathrm{kV}$ gyrotron [3]. Selective excitation at the third harmonic was achieved at a magnetic field close to $10.2 \mathrm{~T}$. The output rf signal with a power of $100-250 \mathrm{~W}$ at a frequency of $0.74 \mathrm{THz}$ corresponded to the transverse mode $\mathrm{TE}_{3,5}$. According to simulations, the share of the ohmic losses in this experiment was relatively low (20\%-25\% of the rf wave power emitted from the electron beam) as compared to the first regular-cavity experiment under this third-harmonic gyrotron [4].

A different method for decreasing the diffraction Q-factor of the operating gyrotron mode is proposed in works $[5,6]$. This is based on exciting a higher axial mode (possessing a relatively low diffractive Qfactor) in the gyrotron-type regime (i.e. at the electron-wave resonance condition $\omega \approx N \Omega$ without Doppler terms). This method is based on the use of a periodically sectioned cavity; every two sections are separated by a short phase corrector providing the $\pi$ shift of the wave phase (Fig. 2). This approach is used to provide operation at the fourth cyclotron harmonic in the $30 \mathrm{keV} / 0.7 \mathrm{~A} \mathrm{CW}$ large-orbit gyrotron [7] based on a $5 \mathrm{~T}$ cryomagnet. The main scope of this setup is to provide operation at the second, third, and fourth cyclotron harmonics at the frequencies $0.26 \mathrm{THz}, 0.39$ $\mathrm{THz}$, and $0.52 \mathrm{THz}$, respectively, with the output power level of hundreds of Watts. In first experiments, the use of a regular $19 \mathrm{~mm}$ cavity provided selective excitation of the mode $\mathrm{TE}_{2,5}$ at the second harmonic $(0.267 \mathrm{THz})$ and of the mode $\mathrm{TE}_{3,7}$ at the third harmonic $(0.394 \mathrm{THz})$ at slightly different magnetic fields. However, the $0.52 \mathrm{THz}$ fourth-harmonic gyrotron requires a long (50-60 wavelengths) operating cavity, so that the diffraction Q-factor of the lowest axial mode (over 100,000) is much greater than the ohmic Q-factor $(\sim 10,000)$, and the share of ohmic losses exceeds $90 \%$. This leads to as low output efficiency of this gyrotron as $0.2 \%$ We designed a cavity consisting of 5 sections and based on the excitation of the mode $\mathrm{TE}_{4,5}$ with 5 axial variations (Fig. 2). Although the cavity is relatively long ( 90 wavelength), sectioning makes possible to reduce the diffraction Qfactor of the operating wave down to $\sim 30,000$, and to reduce the share of ohmic losses down to $\sim 60 \%$. This provides an increase in the output wave efficiency up to $\sim 1 . \%$ (the output power is $\sim 100 \mathrm{~W}$ ).

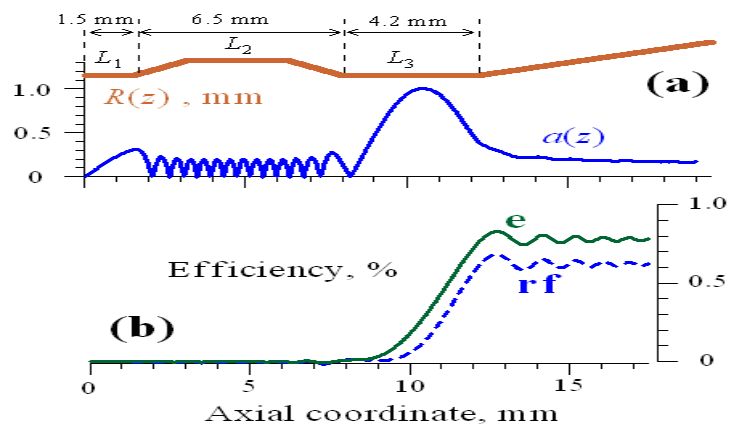

Fig. 1. (a): Sectioned cavity of the third-harmonic largeorbit gyrotron. (b): Results of simulation of the operation at a frequency of $0.74 \mathrm{THz}$; electron efficiency (e) and rf wave efficiency (rf) versus the axial coordinate.

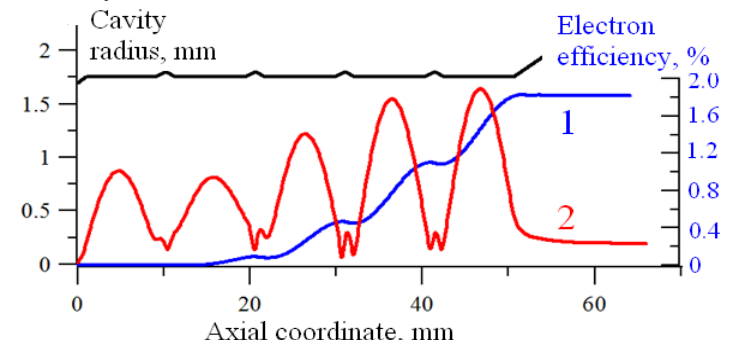

Fig. 2. Large-orbit fourth-harmonic gyrotron: cavity shape, the calculated electron efficiency versus the axial coordinate, and the spatial rf-wave structure of the operating mode corresponding to the $5^{\text {th }}$ axial mode 


\section{Selective irregularities}

A method for suppression of the fundamentalharmonic parasitic waves in second-harmonic gyrotrons based on a simple principle [8] can be used in low-power short-wavelength gyrotrons with a weak electron-wave interaction and, therefore, with long (tens wavelengths) operating cavities. This method is based on the use a quasi-regular cavity with either one or several short irregularities (phase correctors). Evidently, the phase incursion of a near-cutoff wave inside the irregularity is proportional to the frequency, $\phi(\omega) \sim \omega$. For the operating second-harmonic wave, $\phi(\omega)=2 \pi$ and, therefore, such an irregularity does not effect on the excitation of this wave. As for fundamental-harmonic near-cutoff parasitic waves, their phase shift on the same corrector is twice smaller, $\pi$ (Fig. 3 a). The $\pi$-shift of the phase changes the "sign" of the electron-wave coupling (shifts decelerated electron to the accelerating phase of the wave); this lead to a significant degradation of the interaction of electrons with the parasitic waves. This method is valid only for low-mode resonators, when the corrector does not provide scattering of the waves into lower transverse modes. In the case of operation at high radial modes, this approach can be developed by the use of the mode transformation effect (Fig. 3 b) [9]. If the operating high-harmonic mode is $\mathrm{TE}_{\mathrm{m}, \mathrm{n}}$ then the radius of the widening corresponds to the cutoff $\mathrm{TE}_{\mathrm{m}, \mathrm{n}+1}$ wave at the same frequency. Thus, the operating $\mathrm{TE}_{\mathrm{m}, \mathrm{n}}$ wave is converted into the $\mathrm{TE}_{\mathrm{m}, \mathrm{n}+1}$ wave inside the widening with no transformation losses. However, the irregularity is not resonant for the parasitic near-cutoff fundamental-harmonic waves, and a significant reduction of the diffraction Q-factors of these waves is provided due to their scattering. Using this approach, we designed a selective cavity for a pulsed $60 \mathrm{keV}$ gyrotron to achieve second-harmonic excitation of a very developed mode $\left(\mathrm{TE}_{63,15}\right)$ at a frequency of $\sim 1.2 \mathrm{THz}$.

One more approach is based on reducing the cavity length due to the use of a special output irregularity [10]. The irregularity increases the Q-factor of the operating high-harmonic wave due to the reflection, but it almost does not reflect parasitic fundamentalharmonic waves (Fig. $3 \mathrm{c}$ ). In addition, the irregularity spoils the axial structure of the parasitic wave. This approach can provide a significant increase in the starting current of parasitic close-to-cutoff waves with no considerable effect on the generation of the operating high-harmonic wave.

The work is supported by the Russian Science Foundation, project \# 17-19-01605.

\section{References}

1. Savilov, A. V. High-harmonic gyrotron with sectioned cavity // Appl. Phys. Lett. 2009. V. 95, P. 073503.

2. Bandurkin, I. V., Kalynov, Y. K., Savilov, A. V. Highharmonic gyrotron with sectioned cavity // Phys. Plasmas. 2010. V. 17. No. 8. P. 073101.
3. Bandurkin, I. V., Kalynov, Y. K., Savilov, A. V. Experimental realization of the high-harmonic gyrotron oscillator with a klystron-like sectioned cavity // IEEE Trans. Electron Devices. 2015. V. 62. No. 7. P. 2356-2359.

4. Bratman, V. L., Kalynov, Y. K., Manuilov, V. N. Large-orbit gyrotron operation in the terahertz frequency range // Phys. Rev. Lett. 2009. V. 102, No. 24. P. 245101.

5. Bandurkin, I. V., Kalynov, Y. K., Osharin, I. V., Savilov, A. V. Gyrotron with a sectioned cavity based on excitation of a far-from-cutoff operating mode // Phys. Plasmas. 2016. V. 23. N. 1. P. 013113.

6. Bandurkin, I.V., Kalynov, Y.K., Makhalov, P.B., Osharin, I.V., Savilov, A.V., Zheleznov, I.V. Simulations of sectioned cavity for high-harmonic gyrotron // IEEE Trans. Electron Devices. 2017. V. 64 (1). P. 300.

7. Bandurkin, I.V., Bratman, V.L., Kalynov, Y.K., Osharin, I.V., Savilov, A.V. Terahertz large-orbit highharmonic gyrotrons at IAP RAS // IEEE Trans. Electron Devices. 2018. V. 65. P. 2287.

8. Bandurkin, I. V., Kalynov, Y. K., Osharin, I. V., Savilov, A. V. A method for suppression of spurious fundamental-harmonic waves in gyrotrons operating at the second cyclotron harmonic // Phys. Plasmas. 2016. V. 23. No. 5. P. 053116.

9. Bandurkin, I. V., Glyavin, M.Y., Kuzikov, S.V., Makhalov, P.B., Osharin, I. V., Savilov, A. V. Method of providing the high cyclotron harmonic operation selectivity in a gyrotron with a spatially developed operating mode // IEEE Trans. Electron Devices. 2017. V. 64. P. 3893.

11. Oparina, Yu.S., Savilov, A.V. Improvement of mode selectivity of high-harmonic gyrotrons by using opeating cavities with short output reflector // J. IR MM THz Waves 2018. V. 39. P. 595.
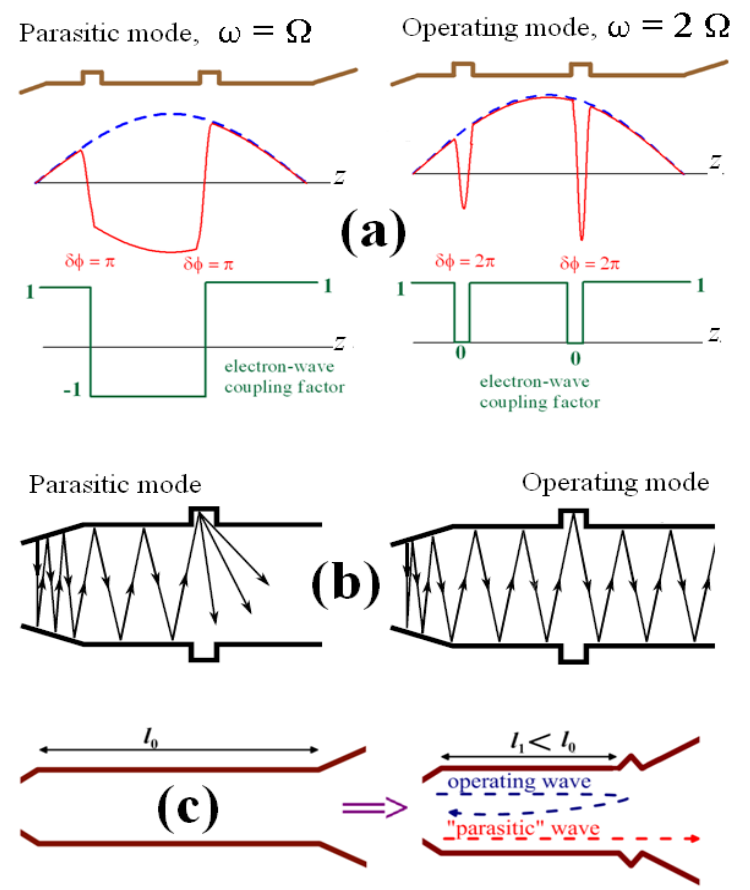

Fig. 3. Gyrotron cavities with selective irregularities. (a): Phase corrector, which provides the $2 \pi$ phase incursion for the operating second-harmonic wave, and the $\pi$ phase incursion for the parasitic fundamental-harmonic wave. (b): An irregularity providing scattering of the parasitic near-cutoff wave. (c): Shortening of the gyrotron operating cavity by the use of the reflecting output irregularity. 